\title{
SPIN-POLARIZED HARTREE-FOCK-SLATER CALCULATIONS IN ATOMS AND DIATOMIC MOLECULES WITH THE FINITE ELEMENT METHOD
}

\author{
D. HEINEMANN, A. ROSÉN \\ Department of Physics, Chalmers University of Technology and University of Göteborg, S-41296 Göteborg, Sweden
} and

B. FRICKE

Department of Physics, University of Kassel, D-3500 Kassel, Federal Republic of Germany

Received 22 November 1989; in final form 14 December 1989

\begin{abstract}
We present spin-polarized Hartree-Fock-Slater calculations performed with the highly accurate numerical finite element method for the atoms $\mathrm{N}$ and $\mathrm{O}$ and the diatomic radical $\mathrm{OH}$ as examples.
\end{abstract}

In recent years there has been an increasing interest in the use of numerical methods for the self-consistent solution of the Hartree-Fock-Slater (HFS) equations as a complement to standard basis set oriented approaches [1-3]. Extensive calculations performed with the finite difference as well as with the finite element method (FEM) have shown that these numerical methods are powerful tools to achieve highly accurate results for diatomic molecules. As example, it can be mentioned that calculations for the closed-shell systems $\mathrm{CO}, \mathrm{N}_{2}$ and $\mathrm{BH}$ have been performed with an accuracy of up to $10^{-8}$ au for the total energy [4].

In order to continue the development of the FEM and its application in atomic and molecular physics we study in this work the use of the FEM to solve the spin-polarized HFS equations for open-shell atoms and diatomic molecules. These equations read

$\left[-\frac{1}{2} \nabla^{2}+V^{\sigma}(\boldsymbol{r})\right] \varphi_{i}^{\sigma}(\boldsymbol{r})=\epsilon \varphi_{i}^{\sigma}(\boldsymbol{r})$,

with

$V^{\sigma}(\boldsymbol{r})=V_{\text {nuc }}(\boldsymbol{r})+V_{\mathrm{c}}(\boldsymbol{r})+V_{\mathrm{x}}^{\sigma}(\boldsymbol{r})$,

$V_{\mathrm{nuc}}(\boldsymbol{r})=-\frac{Z_{1}}{\left|\boldsymbol{r}_{1}+\boldsymbol{r}\right|}-\frac{Z_{2}}{\left|\boldsymbol{r}_{2}+\boldsymbol{r}\right|}$

$$
\begin{aligned}
& V_{\mathrm{c}}(\boldsymbol{r})=\int \frac{\rho\left(\boldsymbol{r}^{\prime}\right)}{\left|\boldsymbol{r}-\boldsymbol{r}^{\prime}\right|} \mathrm{d} \boldsymbol{r}^{\prime}, \\
& V_{\mathrm{x}}^{\sigma}(\boldsymbol{r})=-3 \alpha\left[(3 / 4 \pi) \rho^{\sigma}(\boldsymbol{r})\right]^{1 / 3},
\end{aligned}
$$

where $V_{\text {nuc }}(r)$ is the nuclear Coulomb potential of nuclei 1 and 2 with charges $Z_{1}$ and $Z_{2}$ at positions $r_{1}$ and $r_{2}, V_{\mathrm{c}}(\boldsymbol{r})$ is the electronic Coulomb potential and $V_{\mathbf{x}}^{\sigma}(\boldsymbol{r})$ the remaining electron-electron interactions, i.e. for the exchange and correlation contributions. Here the superscript $\sigma$ denotes the spin quantum number, so $\rho^{\sigma}(r)$ is either the charge density of the spin up or the spin down electrons, respectively. $\rho(r)$ is the total electronic charge density. For open-shell systems the spin polarized HFS equations result in different exchange potentials for the spin up and the spin down orbitals. Within this model the total energy $E_{\text {tot }}$ for the molecular system can be calculated as

$$
\begin{gathered}
E_{\mathrm{tot}}=\sum_{i=1}^{N} \boldsymbol{\epsilon}_{i}-\frac{1}{2} \int \rho(\boldsymbol{r}) V_{\mathrm{c}}(\boldsymbol{r}) \mathrm{d} \boldsymbol{r} \\
-\frac{1}{4} \sum_{\sigma} \int \rho^{\sigma}(\boldsymbol{r}) V_{\mathrm{x}}^{\sigma}(\boldsymbol{r}) \mathrm{d} \boldsymbol{r} .
\end{gathered}
$$

The implementation of the FEM to the solution of the spin-polarized HFS equations was done in ex- 
actly the same way as described in ref. [2] for the solution of the non-polarized HFS equations. Compared to a non-polarized calculation, the CPU time used for the solution of the spin-polarized equations is doubled. This is due to the solution of the eigenvalue equations for the spin up and spin down orbitals.

In order to improve the numerical accuracy of the calculations for a given number of points a logarithmic scaling of the sizes of the elements is introduced. The scaling is done by distributing the points for the coordinate $s$ from ref. [2] by the transformation

$s_{i}=s_{\max } \frac{\gamma^{-N}\left(\gamma^{i}-1\right)}{1-\gamma^{-N}}, \quad i=0, \ldots, N$.

Here $N+1$ is the number of points for this coordinate. The scaling factor $\gamma$ was chosen by optimizing the results for the Thomas-Fermi potential. A typical value is $\gamma=1.2$. This approach is not at all optimal, but it improves the accuracy by one order of magnitude compared to equidistant point distributions.

To test this computational approach calculations were done for some atomic systems. In table 1 the results of the atoms $\mathrm{N}$ and $\mathrm{O}$ are given for ${ }^{4} \mathrm{~S}$ and ${ }^{3} \mathrm{P}$ states, respectively. The values for the total energy and eigenvalues agree within $10^{-5}$ au with the results of one-dimensional calculations also given in table 1 . About 260 points were needed to achieve this accuracy for the two atomic systems.

As a first application for diatomic molecules the radical $\mathrm{OH}$ was chosen. Fig. 1 shows the potential energy curve between 1.4 and 5.5 au including a

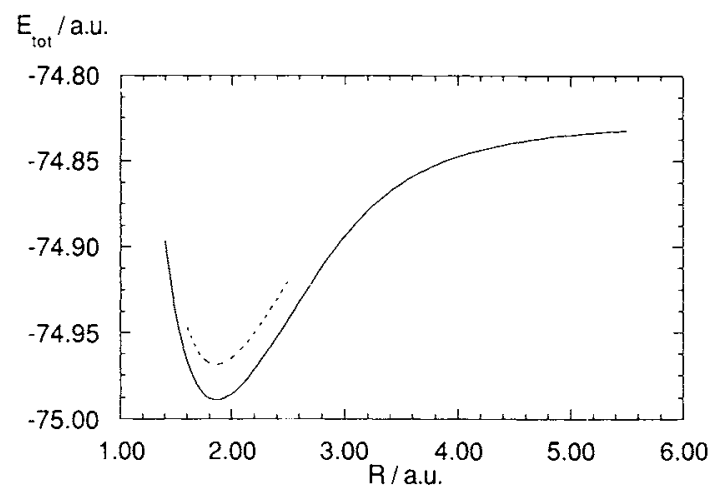

Fig. 1. Comparison of potential energy curves for the $\mathrm{OH}$ radical evaluated with spin polarized (full curve) and non-polarized HFS potential (dotted curve) with $\alpha=0.70$.

comparison with the result from a non-polarized HFS calculation. The numerical accuracy of the total energy is about $10^{-6}$ au over this region of internuclear distances. To achieve this accuracy the calculations were performed with 1369 grid points. We notice how the spin polarized calculations lower the total energy with about $0.02 \mathrm{au}$ in comparison with the non-polarized values.

From the potential energy curve an equilibrium bond length $r_{\mathrm{e}}=1.86$ au and a binding energy $E_{\mathrm{b}}=4.43 \mathrm{eV}$ has been calculated for this system. The agreement with the experimental [5] bond length $r_{\mathrm{e}}=1.83 \mathrm{au}$ and the binding energy $E_{\mathrm{b}}=4.392 \mathrm{eV}$ is good and probably fortuitous. The vibrational energy which was calculated by a least-squares fit of the potential energy curve to a Morse potential gives the value $\omega_{\mathrm{e}}=3714 \mathrm{~cm}^{-1}$, which should be compared to

Table 1

Total energy $E_{\text {tot }}$ and orbital energies $e_{i}$ for the ${ }^{4} \mathrm{~S}$ and ${ }^{3} \mathrm{P}$ state of the atomic systems $\mathrm{N}$ and $\mathrm{O}$. All values are given in au. 1D denotes the one-dimensional calculation

\begin{tabular}{|c|c|c|c|c|}
\hline & \multicolumn{2}{|l|}{$\mathrm{N}$} & \multicolumn{2}{|l|}{0} \\
\hline & $1 \mathrm{D}$ & this work & $1 \mathrm{D}$ & this work \\
\hline$E_{\mathrm{tot}}$ & -54.0018 & -54.00182 & -74.3565 & -74.35651 \\
\hline$e_{\mathrm{isu}}$ & -14.01138 & -14.01139 & -18.79722 & -18.79722 \\
\hline$e_{1 s d}$ & -13.93282 & -13.93282 & -18.73319 & -18.73318 \\
\hline$e_{2 s u}$ & -0.707123 & -0.707122 & -0.898507 & -0.898506 \\
\hline$e_{2 s \mathrm{~d}}$ & -0.490197 & -0.490197 & -0.749675 & -0.749670 \\
\hline$e_{2 \mathrm{pu}}$ & -0.294057 & -0.294057 & -0.362628 & -0.362627 \\
\hline$e_{2 \mathrm{pd}}$ & $-0.093192^{a)}$ & $-0.093192^{a)}$ & -0.221280 & -0.221276 \\
\hline
\end{tabular}

a) Unoccupied level. 
the experimental value of $3737.76 \mathrm{~cm}^{-1}$.

The very good agreement between theory and experiment demonstrates that this method already includes a great part of the correlation energy, but it is far easier to use then all CI-type calculations. Although only one example was presented in this paper, this method might be an appropriate method for a large number of systems in order to study trends for the binding energies, bond length and vibrational frequencies. This is especially interesting for the transition-metal systems with not too high $Z$, but which nevertheless contain a large number of electrons. Further optimizations of the point distributions should make it possible to calculate this kind of system within reasonable computing times. In addition this method opens up the interesting possibility to compare accurate experimental potential energy curves for different internuclear distances with theoretical ones evaluated by the use of different exchange correlation potentials [6].
This work was supported by the Swedish Natural Science Research Council and NORDITA. Computation time at CRAY X-MP/48 from National Supercomputer Centre at University of Linköping is gratefully acknowledged.

\section{References}

[1] L. Laaksonen, D. Sundholm and P. Pyykkö, Computer Phys. Rept. 4 (1986) 313.

[2] D. Heinemann, B. Fricke and D. Kolb, Phys. Rev. A 38 (1988) 4998.

[3] M. Defranceschi and J. Delhalle, eds., Numerical determination of the electronic structure of atoms, diatomic and polyatomic molecules, NATO ASI Series C, Vol. 271 (Kluwer, Dordrecht, 1988).

[4] D. Heinemann, B. Fricke and D. Kolb, Chem. Phys. Letters 145 (1988) 125.

[5] K.P. Huber and G. Herzberg, Constants of diatomic molecules (Van Nostrand Reinhold, New York, 1979).

[6] J.P. Dahl and J. Avery, eds., Local density approximations in quantum chemistry and solid state physics (Plenum Press, New York, 1984) 